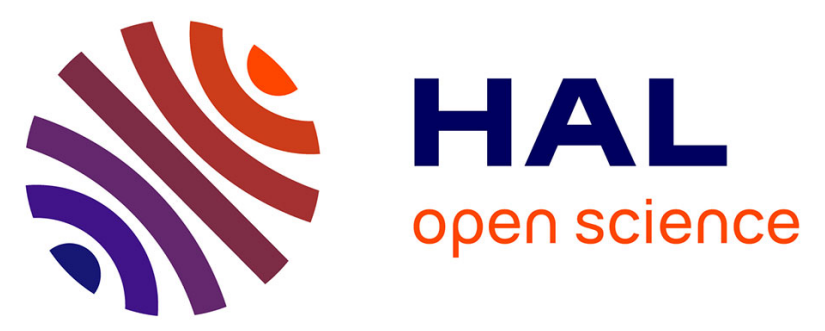

\title{
Coherent dual-frequency emission of a vertical external-cavity semiconductor laser at the cesium D2 line
}

Fabiola A. Camargo, Jessica Barrientos-Barria, Ghaya Baili, Loïc Morvan, Daniel Dolfi, David Holleville, S. Guérandel, Isabelle Sagnes, Patrick Georges, Gaëlle Lucas-Leclin

\section{To cite this version:}

Fabiola A. Camargo, Jessica Barrientos-Barria, Ghaya Baili, Loïc Morvan, Daniel Dolfi, et al.. Coherent dual-frequency emission of a vertical external-cavity semiconductor laser at the cesium D2 line. IEEE Photonics Technology Letters, 2012, 24 (14), pp.1218 - 1220. 10.1109/LPT.2012.2199103 . hal-00713194

\section{HAL Id: hal-00713194 \\ https://hal-iogs.archives-ouvertes.fr/hal-00713194}

Submitted on 29 Jun 2012

HAL is a multi-disciplinary open access archive for the deposit and dissemination of scientific research documents, whether they are published or not. The documents may come from teaching and research institutions in France or abroad, or from public or private research centers.
L'archive ouverte pluridisciplinaire HAL, est destinée au dépôt et à la diffusion de documents scientifiques de niveau recherche, publiés ou non, émanant des établissements d'enseignement et de recherche français ou étrangers, des laboratoires publics ou privés. 


\title{
Coherent dual-frequency emission of a vertical external-cavity semiconductor laser at the cesium $\mathrm{D}_{2}$ line
}

\author{
F. A. Camargo, J. Barrientos, G. Baili, L. Morvan, D. Dolfi, \\ D. Holleville, S. Guerandel, I. Sagnes, P. Georges, G. Lucas-Leclin
}

\begin{abstract}
We describe the dual-frequency and dual-polarization emission of a diode-pumped vertical external-cavity semiconductor laser at $852 \mathrm{~nm}$ dedicated to the coherent population trapping of cesium atoms. The output power reaches $\sim 20 \mathrm{~mW}$ on each frequency, with a frequency difference in the $\mathrm{GHz}$ range.
\end{abstract}

Index Terms - metrology, semiconductor lasers, vertical cavity surface emitting lasers

\section{INTRODUCTION}

$\mathrm{C}$ oherent population trapping (CPT) has been demonstrated as an interesting technique for miniature atomic frequency references and quantum information [1]. It is based on the coupling of the two hyperfine ground states of an alkali atom - namely cesium $\left({ }^{133} \mathrm{Cs}\right)$ for atomic clocks - through excitation to a common atomic level by two phase-coherent laser fields nearly resonant with the atomic transitions. The frequency difference between the two laser fields is tuned at the atomic frequency splitting in the microwave range, equal to $9.192 \mathrm{GHz}$ for ${ }^{133} \mathrm{Cs}$ atoms. Output power in the $\mathrm{mW}$ range and narrow-linewidth emission $(<500 \mathrm{kHz})$ are required for the two laser beams. The common solutions for the realization of the coherent pair of laser beams are sideband generation by microwave modulation of a single-frequency diode laser [2] and electronic phase-locking of two independent lasers [3]. Though the latter configuration is ideal from a metrological point of view, it requires a complex and costly wideband phase-locked electronics loop. As an alternative to these solutions, we propose the dual-frequency optically-pumped vertical-external-cavity semiconductor laser (OP-VECSEL), based on the simultaneous oscillation of two cross-polarized longitudinal modes in the same laser cavity. This configuration has been primarily investigated for the

Manuscript received February 8, 2012. This work was supported by the French Agence Nationale de la Recherche (ANR-07-BLAN-0320-03). F. Camargo was funded by RTRA/Triangle de la Physique (2010-089T).

F. A. Camargo, J. Barrientos, P. Georges, G. Lucas-Leclin are with the Laboratoire Charles Fabry, Institut d'Optique, CNRS, Univ Paris-Sud XI, 91127 Palaiseau, France (corresponding author e-mail: gaelle.lucas-leclin@ institutoptique.fr).

G. Baili, L. Morvan, D. Dolfi are with Thales Research \& Technology, 91767 Palaiseau, France.

S. Guérandel, D. Holleville are with LNE-SYRTE, Systèmes de Référence Temps-Espace, Observatoire de Paris, CNRS, UPMC, 75014 Paris (France)

I. Sagnes is with the Laboratoire de Photonique et de Nanostructures, CNRS, 91460 Marcoussis (France)

Copyright (C) 2012 IEEE. generation of tunable optically-carried microwave sources; it has been demonstrated with diode-pumped solid-state lasers [4], and recently with an OP-VECSEL operating at 1 $\mu \mathrm{m}[5]$.

Actually dual-frequency laser operation of an OP-VECSEL presents many advantages: first, the two laser fields experience strongly correlated frequency and intensity fluctuations, as they share the same cavity; second, the frequency difference, which is proportional to the intracavity phase anisotropy, may be tunable from few tens of $\mathrm{MHz}$ to a few THz. Additionally, centimeter-long-cavity OP-VECSEL do not present relaxation oscillations thanks to a class-A dynamical behavior, which results from a photon lifetime inside the laser cavity longer than the excited-carriers lifetime [6]. Thus the relative-intensity-noise (RIN) is low, shot-noise limited on a wide spectral range. Finally, single-frequency operation of OP-VECSEL with narrow spectral linewidth has already been established [7]. Dual-frequency OP-VECSEL emitting at the wavelength of a Cs transition would thus be an innovating solution with major potentials for miniature atomic clocks.

In this work, we report for the first time the dual-frequency and dual-polarization operation of an OP-VECSEL emitting at $852.1 \mathrm{~nm}$, with $\sim 20 \mathrm{~mW}$ power in each polarization.

\section{EXPERIMENTAL SETUP}

The semiconductor structure is designed for an emission at $\lambda_{L}=852 \mathrm{~nm}$ under barrier-pumping at $\lambda \leq 700 \mathrm{~nm}$. The $30 \lambda / 4$-thick active region is composed of seven 8 -nm thick GaAs quantum wells, embedded between $\mathrm{Al}_{0.22} \mathrm{Ga}_{0.78} \mathrm{As}$ barriers [8]. The bottom Distributed Bragg Reflector (DBR) consists of 32.5 pairs of $\mathrm{AlAs} / \mathrm{Al}_{0.22} \mathrm{Ga}_{0.78} \mathrm{As} \lambda / 4$-thick layers, and is reflectivity is evaluated to $\mathrm{R}>99.95 \%$ at 852 $\mathrm{nm}$. The active structure is grown on a $350 \mu \mathrm{m}$-thick GaAs substrate. A $20 \mathrm{~nm}$-thick $\operatorname{In}_{0.48} \mathrm{Ga}_{0.52} \mathrm{P}$ top layer protects the structure from oxidation; a $\lambda / 4$-thick $\mathrm{Si}_{3} \mathrm{~N}_{4}$ anti-reflection coating suppresses the Fabry-Perot resonance of the semiconductor chip and increases the pump transmission. With a similar chip, more than $300 \mathrm{~mW}$ was already obtained under $1 \mathrm{~W}$-pumping at $T=0{ }^{\circ} \mathrm{C}$ [8].

The pump source is $2 \mathrm{~W}$-broad-area laser diode emitting at $670 \mathrm{~nm}$ coupled into a $100 \mu \mathrm{m}$ diameter, $\mathrm{NA}=0.22$, multimode fiber. The optimal beam size regarding the output power was measured to be $\sim 80 \mu \mathrm{m}$ for this structure [8]. For this reason the pump beam was imaged with two aspherical lenses of $f_{1}=11 \mathrm{~mm}$ and $f_{2}=8 \mathrm{~mm}$ under a $45^{\circ}$ 
angle yielding an $80 \mu \mathrm{m} \times 120 \mu \mathrm{m}$-elliptical spot on the structure.

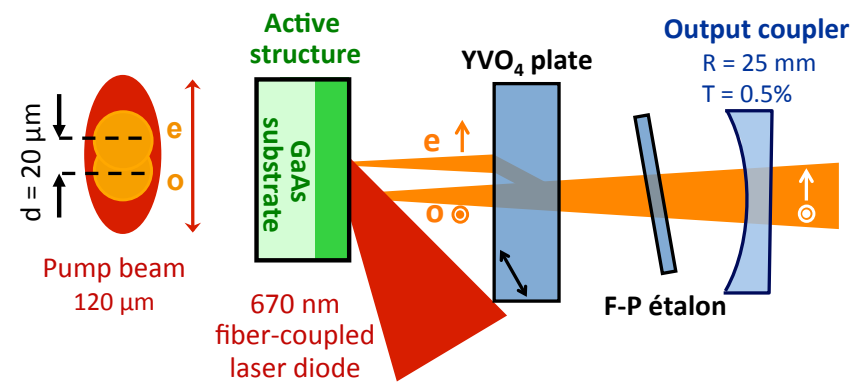

Fig. 1. Experimental setup for the OP-VECSEL (right) and the laser and pump beam superposition on the chip surface (left).

The laser cavity design has focused on compactness as well as high mechanical and thermal stability. The cavity length is $L \cong 24 \mathrm{~mm}$, resulting in a free spectral range $F S R_{C}$ $=6 \mathrm{GHz}=14 \mathrm{pm}$. The output coupler is a spherical mirror with a radius of curvature $R_{c}=25 \mathrm{~mm}$ and a transmission $T$ $=0.5 \%$ at $852 \mathrm{~nm}$ (Fig. 1). It is glued on a piezo-electric transducer (PZT) for the fine adjustment of the cavity length. The laser beam diameter is $\sim 70 \mu \mathrm{m}$ in the structure, which allows a good overlap between the pump and the laser beam. The semiconductor chip is fixed on a thermoelectric TO3-like module, whose temperature is controlled at $15^{\circ} \mathrm{C}$. The pump optics, the semiconductor chip and the laser cavity elements are integrated in a compact $70 \mathrm{~mm} \times 70 \mathrm{~mm} \times 60 \mathrm{~mm}$ aluminium casing. This effectively limits mechanical and acoustic vibrations as well as air temperature fluctuations inside the external cavity. The temperature of the whole setup is stabilized to $15^{\circ} \mathrm{C}$ with a Peltier element.

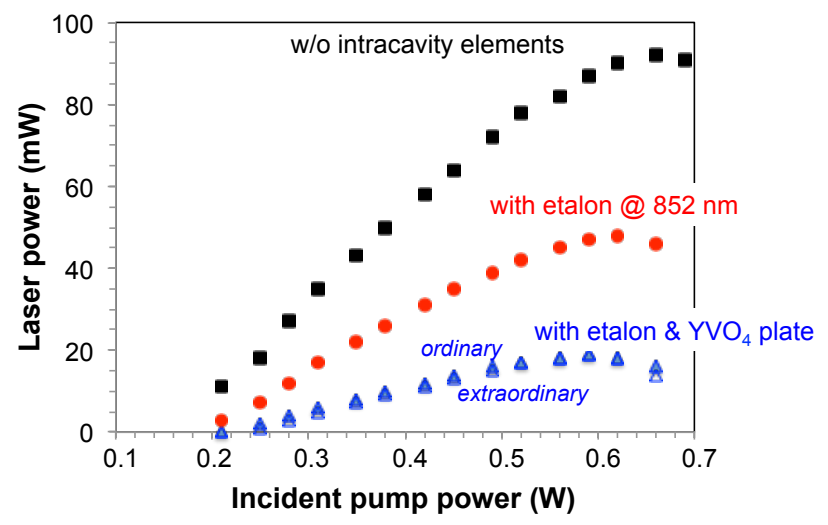

Fig. 2. Laser output power versus the incident pump power without any intracavity element (black squares), under single-frequency at $852 \mathrm{~nm}$ with the intracavity etalon (red circles) and under 2-frequency operation at 852 $\mathrm{nm}$ with the etalon and the $\mathrm{YVO}_{4}$ plate (blue triangles).

Without any intracavity element, the laser oscillates in multi-longitudinal modes around $\lambda=856 \mathrm{~nm}$; the laser threshold is $0.15 \mathrm{~W}$, and the output power reaches $92 \mathrm{~mW}$ for an incident pump power of $0.66 \mathrm{~W}$, limited by the thermal roll-over of the active structure (Fig. 2). Wavelength-tunable single-frequency operation of the laser is obtained with an intracavity $25 \mu \mathrm{m}$-thick silica etalon $\left(F S R_{E} \cong 4 \mathrm{THz}=10 \mathrm{~nm}\right)$. Due to higher intracavity losses, the laser threshold increases to $0.17 \mathrm{~W}$. The output power decreases to $68 \mathrm{~mW}$ for single-frequency emission at 856 $\mathrm{nm}$, and $48 \mathrm{~mW}$ at $852 \mathrm{~nm}$. It is noteworthy that in this configuration, the laser emission is linearly polarized, parallel to the long axis of the pump ellipse due to the slight pump-induced gain anisotropy of the semiconductor active region. The output beam is circular and single transverse mode.

\section{DUAL-FREQUENCY EMISSION LASER}

The principle of the dual-frequency emission of the VECSEL lies on a controlled phase anisotropy inside the laser cavity [4]. In our experiment, a $200 \mu \mathrm{m}$-thick, antireflection coated, birefringent $\mathrm{YVO}_{4}$ plate cut at $45^{\circ}$ to its optic axis is introduced in the laser cavity between the Fabry-Perot etalon and the active structure (Fig. 1). The phase anisotropy of the $\mathrm{YVO}_{4}$ plate results in two different cavity lengths, respectively for the ordinary and extraordinary polarizations, and subsequently in two different combs of longitudinal modes. Additionally, the $\mathrm{YVO}_{4}$ plate separates the cross-polarized laser beams from a distance $d=20 \mu \mathrm{m}$ on the active structure, in the longer axis of the pump ellipse. This separation reduces the nonlinear coupling between the two modes and ensures a stable dual-frequency dual-polarization emission [9]; nevertheless it is small enough to maintain a good overlap between the pump beam and the two laser spots (Fig 1.). Still, the cross-polarized beams share most of the cavity length, and thus experience the same mechanical and thermal fluctuations, which should result in an inherent high-purity beat-note in the microwave range. As the birefringent plate introduces additional losses in the laser cavity, the laser threshold increases to $0.21 \mathrm{~W}$. The laser emission is purely single-frequency on each polarization, with equal output powers of $19 \mathrm{~mW}$ at the incident pump power of $0.6 \mathrm{~W}$ (Fig. 2).

The frequency difference $\Delta v$ between the two cross-polarized laser lines is directly related to the free spectral range of the laser cavity $F S R_{C}$, the $\mathrm{YVO}_{4}$ plate thickness $e$ and the intracavity phase anisotropy, and can be approximated by

$$
\Delta v=v_{e}-v_{o} \cong \frac{\Delta n \times e}{L} \frac{c}{\lambda} \pm k \times F S R_{C}
$$

where $c$ is the light velocity, $\Delta n$ is the difference between the refractive indices at each polarization, $L$ is the laser cavity length, $\lambda$ is the laser wavelength and $k$ is an integer. The two cross-polarized modes oscillate at frequencies corresponding to their lowest roundtrip optical losses. As the spectral selectivity $F S R_{E}$ of the Fabry-Perot etalon is much broader than the distance between cavity modes $F S R_{C}$, the cross-polarized modes may oscillate a few $F S R_{C}$ apart. The actual frequency difference is not known a priori, but can be adjusted by a fine adjustment of the etalon angle, and by tilting the $\mathrm{YVO}_{4}$ plate taking benefit of the dependency of the $\mathrm{YVO}_{4}$ birefringence with the incidence angle. Experimentally, the frequency difference $\Delta v$ takes discrete values, from few $\mathrm{GHz}$ to hundreds of $\mathrm{GHz}$. It is continuously tuned over a few $\mathrm{MHz}$ with the cavity length. Figure 3 (top) shows the high-resolution optical spectrum of the dual-frequency laser in a configuration for which $\Delta v$ is smaller than the FSR of the laser cavity; thus the laser operates on two adjacent cross-polarized modes. The dual-frequency emission is also evidenced in the RF beat-note spectrum obtained by mixing the cross-polarized lines with a polarizer oriented at $45^{\circ}$ of their axes and focusing the laser beam onto an ultrafast InGaAs MSM photodetector followed by a RF amplifier (Fig. 3 bottom). The beat-note spectrum width, measured on a electrical spectrum analyzer, is $\sim 150 \mathrm{kHz}$ (FWHM), limited by 
residual mechanical vibrations of the birefringent plate which induce a jitter of the beat-note frequency over the 100 $\mathrm{ms}$ averaging time (Fig.3). From similar experiments [5][10], we expect the instantaneous beat-note width to be below $4 \mathrm{kHz}$ under free-running operation, and below $1 \mathrm{~Hz}$ with a phase-locking loop on a microwave reference. Even without active stabilization of the cavity length, the beat-note frequency drift remains below $500 \mathrm{kHz}$ on a $1 \mathrm{mn}$ time thanks to the stability of our mechanical setup.
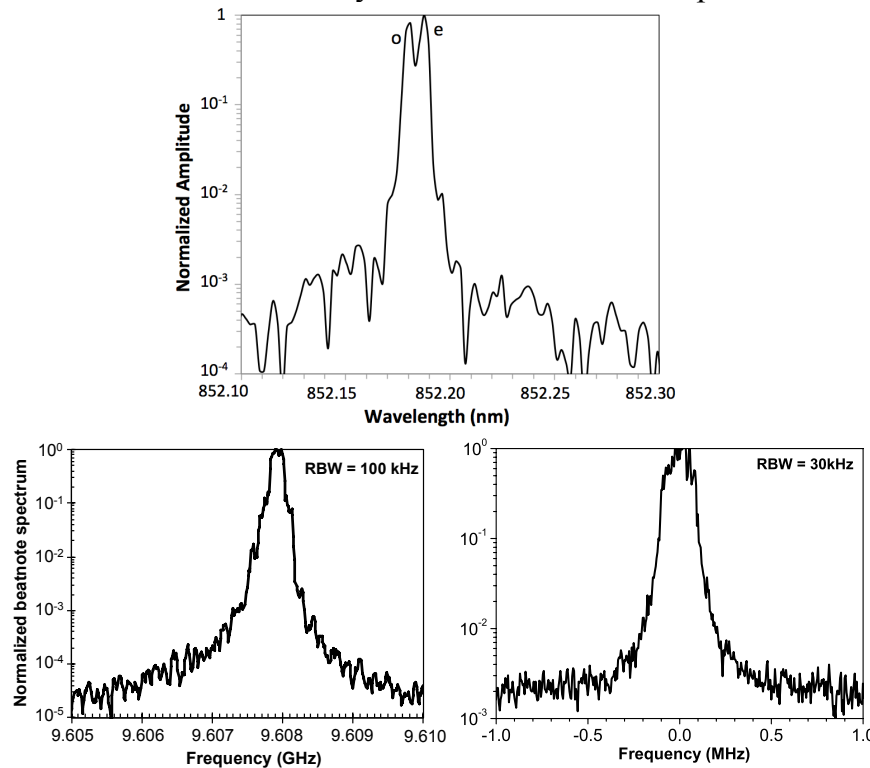

Fig. 3. (top) Dual-frequency laser spectrum measured with a Fourier transform optical analyzer (resolution $=2 \mathrm{pm}$ ); (bottom) Averaged RF beat-note spectra with different resolution bandwidths (left : RBW $=100 \mathrm{kHz}$; right : $\mathrm{RBW}=30 \mathrm{kHz}$ ).

Finally, the laser line has been stabilized onto an atomic hyperfine transition of the $\mathrm{Cs}_{2}$ line at $852.14 \mathrm{~nm}$; the laser frequency at the ordinary polarization has been locked to the side of a Doppler-free saturated absorption line through a low-bandwidth $(f<1 \mathrm{kHz})$ servo loop on the piezoelectric transducer glued onto the output coupler. The root-mean-square (RMS) frequency error signal is $<500$ $\mathrm{kHz}$ on $100 \mathrm{~ms}$, which is an evaluation of the global frequency fluctuations of the laser frequency relatively to the atomic reference. It is comparable to our previous results under single-frequency emission with a single-transverse-mode pump laser source [8], making us confident that the laser linewidth on each polarization would be compatible with the requirements for the CPT atomic clock experiment. This is further confirmed by the narrow linewidth of the Doppler-free absorption lines excited by the laser. Though the laser cavity length was stabilized, the RF beat-note linewidth remains unchanged, which confirmed that it is principally limited by the birefringence instabilities within the laser cavity.

\section{CONCLUSION}

We have demonstrated the dual-frequency and dual-polarization operation of a diode-pumped vertical-external-cavity semiconductor laser emitting at the Cs $\mathrm{D}_{2}$ line at $852 \mathrm{~nm}$, with a frequency difference in the microwave range. The output power at each frequency reaches $19 \mathrm{~mW}$. In this experiment, the frequency difference $\Delta v$ between the two cross-polarized beams was fixed by the birefringent component; in the next future, the addition of an intracavity electro-optic modulator will allow to phase-lock the frequency difference $\Delta v$ at $9.2 \mathrm{GHz}$ and then stabilize to Cs hyperfine splitting. This work is thus the first step towards the realization of a new laser source for the production of a coherent pair of tunable optical frequencies dedicated to CPT atomic clocks.

\section{ACKNOWLEDGMENTS}

The authors acknowledge A. Garnache, from Institut d'Electronique du Sud (Montpellier, France), for the design of the active structure and fruitful discussions on OP-VECSELs, and F. Bretenaker, from Laboratoire Aimé Cotton (Palaiseau, France), for his contribution to dual-frequency laser cavity designs. They are also grateful to B. Sumpf and G. Erbert, from Ferdinand-Braun Institut für Höchstfrequenztechnik (Berlin, Germany) who provided the pump laser source.

\section{REFERENCES}

[1] J. Kitching, S. Knappe, N. Vukicevic, L. Hollberg, R. Wynands, and W. Weidmann, "A Microwave Frequency Reference Based on VCSEL-Driven Dark Line Resonances in Cs Vapor", IEEE Trans. Instr. Meas., vol. 49, no. 6, pp 1313-1317, Dec. 2000

[2] M. Merimaa, T. Lindvall, I. Tittonen, and E. Ikonen, "All-optical atomic clock based on coherent population trapping in ${ }^{85} \mathrm{Rb}$," J. Opt. Soc. Amer. B, Opt. Phys., vol. 20, no. 2, pp. 273-279, Feb. 2003

[3] T. Zanon, S. Guerandel, E. de Clercq, D. Holleville, N. Dimarcq, and A. Clairon, "High Contrast Ramsey Fringes with Coherent-Population-Trapping Pulses in a Double Lambda Atomic System", Phys. Rev. Lett. 94, 193002, May 2005

[4] M. Brunel, F. Bretenaker, and A. Le Floch, "Tunable optical microwave sources using spatially resolved laser eigenstates", Opt. Lett., vol. 22, pp 384-387, March 1997

[5] G. Baili, L. Morvan, M. Alouini, D. Dolfi, F. Bretenaker, I. Sagnes and A. Garnache, "Experimental demonstration of a tunable dual-frequency semiconductor laser free of relaxation oscillations", Opt. Lett., vol. 34, pp. 3421-3423, Nov. 2009.

[6] G. Baili, M. Alouini, D. Dolfi, F. Bretenaker, I. Sagnes, A. Garnache, "Shot-noise-limited operation of a monomode high-cavity-finesse semiconductor laser for microwave photonics applications", Opt. Lett. 32, 650 (2007)

[7] B. Rösener, S. Kaspar, M.Rattunde, T. Töpper, C. Manz, K. Köhler, O. Ambacher, J. Wagner, " $2 \mu \mathrm{m}$ semiconductor disk laser with a heterodyne linewidth below $10 \mathrm{kHz} "$, Opt. Lett. 36, 3587 (2011)

[8] B. Cocquelin, D. Holleville, G. Lucas-Leclin, I. Sagnes, A. Garnache, M. Myara and P. Georges, "Tunable single-frequency operation of a diode-pumped vertical external-cavity laser at the cesium $\mathrm{D}_{2}$ line", Appl. Phys. B, vol. 95, pp. 315-321, Jan. 2009

[9] V. Pal, P. Trofimoff, B.-X. Miranda, G. Baili, M. Alouini, L. Morvan, D. Dolfi, F. Goldfarb, I. Sagnes, R. Ghosh and F. Bretenaker, "Measurement of the coupling constant in a two-frequency VECSEL", Opt. Exp., vol. 18, pp. 5008-5014, Mar. 2010.

[10] G. Pillet, L. Morvan, M. Brunel, F. Bretenaker, D. Dolfi, M. Vallet, J.-P. Huignard, A. Le Floch, "Dual-frequency laser at $1.5 \mu \mathrm{m}$ for optical distribution and generation of high-purity microwave signals", J. Light. Techn., vol. 26, 15, 2764-2773 (2008) 